\title{
CoFe-Cu granular alloys: From noninteracting particles to magnetic percolation
}

\author{
Victor Franco, ${ }^{\text {a) }}$ Xavier Batlle, and Amílcar Labarta ${ }^{\text {b) }}$ \\ Departamento de Física Fundamental, Facultad de Física, Universidad de Barcelona, Diagonal, 647, \\ 08028 Barcelona, Spain
}

(Received 27 October 1998; accepted for publication 1 February 1999)

$\mathrm{CoFe}-\mathrm{Cu}$ granular films with ferromagnetic content ranging from 0.10 to 0.33 by volume were prepared by radio frequency sputtering. As-cast samples were rapidly annealed at various temperatures up to $750^{\circ} \mathrm{C}$ to promote the segregation of $\mathrm{CoFe}$ particles within the metallic matrix. Magnetic and transport properties suggested that this family of samples may be classified into three groups: (i) below about 0.20 volume content of $\mathrm{CoFe}$, all samples display the typical features of a granular solid constituted by a random distribution of nanometric $\mathrm{CoFe}$ particles within a $\mathrm{Cu}$ matrix, and the maximum magnetoresistance is about $20 \%$ at low temperature (giant magnetoresistance); (ii) for as-cast samples within 0.20 and 0.30 of volume concentration, magnetoresistance and magnetization display complex bimodal behavior and large metastable effects associated with the interparticle interactions, which stabilize a domain-like microstructure well below the volume percolation threshold $(0.55)$, as already observed in $\mathrm{CoFe}-\mathrm{Ag}(\mathrm{Cu})$ granular alloys. As a consequence of the large magnetic correlations, magnetoresistance is very low $(1 \%-3 \%)$. Through annealing, the microstructure and therefore the transport properties evolve to those of a classical giant magnetoresistance system with large particles; and (iii) above about 0.30 of volume content (and still below the volume percolation threshold), as-cast samples display both anisotropic and giant magnetoresistance, as also observed in other granular alloys. Annealing leads to complete segregation and to the formation of large magnetic particles, which results in a transition from mixed behavior of both anisotropic and giant magnetoresistance (GMR) regimes to a giant magnetoresistance regime, with a maximum GMR of about 7\%. (C) 1999 American Institute of Physics. [S0021-8979(99)01810-1]

\section{INTRODUCTION}

Nanostructured magnetic materials have been largely studied because of both their technological applications and their new challenging magnetic and transport properties. This is the case of granular magnetic alloys consisting on an ultrafine ferromagnetic (FM) particle distribution embedded in a nonmagnetic metallic matrix, which have been extensively studied in the past few years due to their anomalous magnetotransport properties. In particular, because they display negative giant magnetoresistance ${ }^{1}$ (GMR). In these materials, GMR is interpreted within the scope of the spindependent scattering of conduction electrons with the local magnetic configuration ${ }^{2}$ either within or at the interfaces of the FM particles embedded throughout the matrix. Some of these materials also show classical anisotropic magnetoresistance (AMR) ${ }^{3,4}$ In this case, the sign of the magnetoresistance depends on the angle formed between the applied field and the electrical current.

The purpose of this article is to discuss the physical and magnetic microstructure and the magnetic and transport properties of $\mathrm{CoFe}-\mathrm{Cu}$ samples with a volume concentration of $\mathrm{CoFe}\left(x_{v}\right)$ ranging from $x_{v}=0.10$ to $x_{v}=0.33$. Fe was

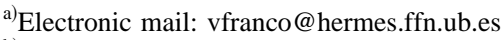

b) Author to whom correspondence should be addressed; electronic mail: amilcar@hermes.ffn.ub.es
}

added to Co (about 5-10 at.\%) in the classical CoCu GMR system as a minor FM component, in order to increase the GMR effect as previously reported, ${ }^{5}$ by increasing the magnetic moment of the particles. Although the physical microstructure is almost the same for all the as-cast samples below the percolation threshold, magnetic and magnetotransport properties change dramatically with $x_{v}$. Furthermore, the annealing procedure greatly modifies both the crystal structure and the magnetic microstructure, and consequently the magnetotransport properties. Finally, it is shown that the observed behavior in $\mathrm{CoFe}-\mathrm{Cu}$ alloys is due to the interplay between: (i) dipolar interactions between magnetic entities closely dispersed in the metallic matrix, (ii) indirect ferromagnetic exchange through the metallic matrix due to $\mathrm{CoFe}$ alloying, which disappears with the annealing, and (iii) outof-plane uniaxial magnetic anisotropy arising from crystal lattice strains, which relaxes and also disappears as a result of annealing.

\section{EXPERIMENT}

$\mathrm{CoFe}-\mathrm{Cu}$ thin films with thickness of about $250 \mathrm{~nm}$ were radio frequency sputtered onto glass microscope slides. The film thickness was measured using a Tolansky multiplebeam interferometer and the composition was determined using energy dispersive $\mathrm{x}$-ray spectrometry and ionic phase chemical mass spectrometry. The as-cast samples were rap- 


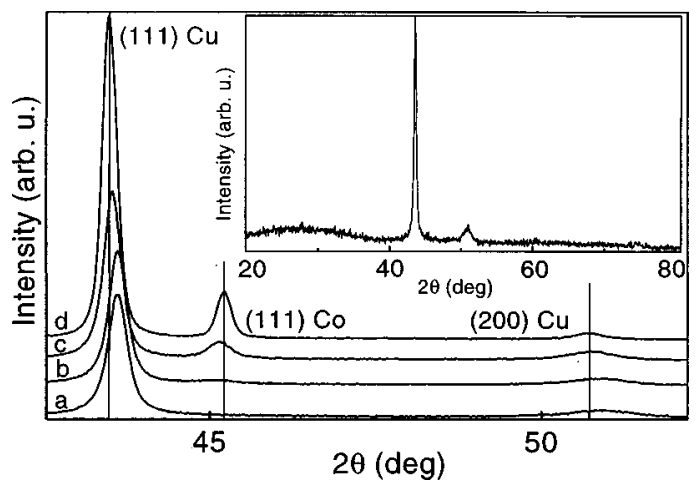

FIG. 1. [(a) and inset] XRD spectra for a $\mathrm{Co}_{23} \mathrm{Fe}_{11} \mathrm{Cu}_{66}$ sample as-cast and annealed at (b) 600 , (c) 650 , and (d) $750{ }^{\circ} \mathrm{C}$. Vertical lines represent bulk values for (111) $\mathrm{Cu}$, (111) $\mathrm{Co}$, and (200) $\mathrm{Cu}$ reflections, respectively.

idly annealed $(0.1 \mathrm{~s})$ at 600,650 , and $750^{\circ} \mathrm{C}$ in a vacuum system, in order to promote phase segregation, particle growth, and strain relaxation. The particle size distribution and microstructure were studied by x-ray diffraction (XRD), atomic force microscopy (AFM), and transmission electron microscopy (TEM). The texture of the samples was studied through the XRD rocking curves, where the full width at half maximum (FWHM) indicates the angular dispersion of a given Bragg reflection. XRD rocking curves give information on the in-plane crystalline quality, such as the lateral structural coherence length and the mosaic spread within the epitaxial film. Magnetic force microscopy (MFM) was used to study the microscopic magnetic pole distribution perpendicular to the film plane. A magnetic tip consisting of a single Si crystal coated with a thin CoCr film (coercive field, $H_{c}=365 \pm 9 \mathrm{Oe}$ ) was oscillated $50 \mathrm{~nm}$ above the film surface. Magnetoresistance (MR) was measured by an alternating current four-point probe technique in the temperature range $2-300 \mathrm{~K}$ and in magnetic fields up to $10 \mathrm{kOe}$. The magnetic field was applied in the film plane parallel to the current direction. Hysteresis loops were recorded with a vibrating sample magnetometer up to $12 \mathrm{kOe}$ and with a superconducting quantum interference device magnetometer up to $50 \mathrm{kOe}$.

\section{RESULTS}

\section{A. Crystal structure and sample texture}

The $\theta / 2 \theta$ XRD spectra for the as-cast samples clearly show the (111) $\mathrm{Cu}$ peak at all the concentrations studied. Other reflections of the $\mathrm{Cu}$ face-centered-cubic (fcc) structure, such as the (222) and the (200) can also be seen, but their intensities are much lower (see Fig. 1 and inset as an example). The spectrum of the amorphous substrate (glass) is observed at low angles (between $0^{\circ}$ and $20^{\circ}$ ), and between $20^{\circ}$ and $40^{\circ}$ the amorphous phase of the metallic alloy is also observed. These results indicate that the as-cast samples have a low degree of crystallinity, because of the rapid quenching produced during deposition. Besides, the $\mathrm{Cu}$ crystallites are mostly oriented along the $\langle 111\rangle$ direction, which is perpendicular to the film plane. However, this texture is not complete since a small peak corresponding to the (200) $\mathrm{Cu}$ reflection is also present. The rocking curves for the (111) $\mathrm{Cu}$
TABLE I. FWHM of the rocking curves for the (111) $\mathrm{Cu}$ and (111) Co reflections for samples $\mathrm{Co}_{11} \mathrm{Fe}_{6} \mathrm{Cu}_{83}$ and $\mathrm{Co}_{23} \mathrm{Fe}_{11} \mathrm{Cu}_{66}$, as-cast and at three annealing temperatures (in brackets).

\begin{tabular}{ccc}
\hline \hline Sample & $(111) \mathrm{Cu}$ & $(111) \mathrm{Co}$ \\
\hline $\mathrm{Co}_{11} \mathrm{Fe}_{6} \mathrm{Cu}_{83}$ as-cast & $26.6^{\circ}$ & $\ldots$ \\
$\mathrm{Co}_{11} \mathrm{Fe}_{6} \mathrm{Cu}_{83}\left(600^{\circ} \mathrm{C}\right)$ & $21.2^{\circ}$ & $29.8^{\circ}$ \\
$\mathrm{Co}_{11} \mathrm{Fe}_{6} \mathrm{Cu}_{83}\left(650^{\circ} \mathrm{C}\right)$ & $19.5^{\circ}$ & $23.4^{\circ}$ \\
$\mathrm{Co}_{11} \mathrm{Fe}_{6} \mathrm{Cu}_{83}\left(750^{\circ} \mathrm{C}\right)$ & $17.9^{\circ}$ & $21.2^{\circ}$ \\
$\mathrm{Co}_{23} \mathrm{Fe}_{11} \mathrm{Cu}_{66}$ as-cast & $35.1^{\circ}$ & $\ldots$ \\
$\mathrm{Co}_{23} \mathrm{Fe}_{11} \mathrm{Cu}_{66}\left(600^{\circ} \mathrm{C}\right)$ & $24.9^{\circ}$ & $32.1^{\circ}$ \\
$\mathrm{Co}_{23} \mathrm{Fe}_{11} \mathrm{Cu}_{66}\left(650^{\circ} \mathrm{C}\right)$ & $17.8^{\circ}$ & $24.4^{\circ}$ \\
$\mathrm{Co}_{23} \mathrm{Fe}_{11} \mathrm{Cu}_{66}\left(750^{\circ} \mathrm{C}\right)$ & $16.1^{\circ}$ & $22.3^{\circ}$ \\
\hline \hline
\end{tabular}

peak for $\mathrm{Co}_{11} \mathrm{Fe}_{6} \mathrm{Cu}_{83}$ show an angular dispersion (with respect to the film normal) of $26.6^{\circ}$ and $17.9^{\circ}$ for the as-cast and $750^{\circ} \mathrm{C}$ annealed samples, respectively. The degree of texture increases slightly with both $\mathrm{Cu}$ concentration and annealing temperature, as expected (see Table I). CoFe forms a fcc solid solution at all the concentrations studied. These crystals, display similar texture although the peaks are weaker since the degree of crystallinity is lower. In the ascast samples, CoFe crystals are not large (or well crystallized) enough to be easily observed in conventional $\theta / 2 \theta$ spectra. Specially detailed spectra were recorded to locate the CoFe peaks position. This is also supported by the fact that magnetic and transport properties are closer to those of a $\mathrm{CoFeCu}$ alloy. To verify the fcc structure and the texture of the samples, other $\theta / 2 \theta$ spectra were recorded at different $\chi$ angles (by tilting the sample with respect to an axis parallel to the film plane), in such a manner that by rotating the sample $35^{\circ}$ (expected angle between the $\langle 111\rangle$ and the $\langle 100\rangle$ cube directions), the relative intensity of the (200) Cu reflection becomes maximal with respect to the (111) reflection, confirming the sample texture and crystal structure (Fig. 2). All the peaks are shifted from their corresponding bulk values (Fig. 1) because of strains during deposition due to the differences between thermal expansion coefficients of the glass substrate and the sample film, and to the presence of metal coherent interfaces (MCIs) and $\mathrm{CoFe}-\mathrm{Cu}$ alloying. As shown by the shifting of the peaks, the lattice spacings $d_{h k l}$ of the bulk fcc $\mathrm{CoFe}$ and $\mathrm{Cu}$ are modified. Furthermore, $d_{111}$ is shorter than expected, while the $d_{200}$ is longer (see Fig. 3). This leads to a deformed cubic cell squeezed in the direction

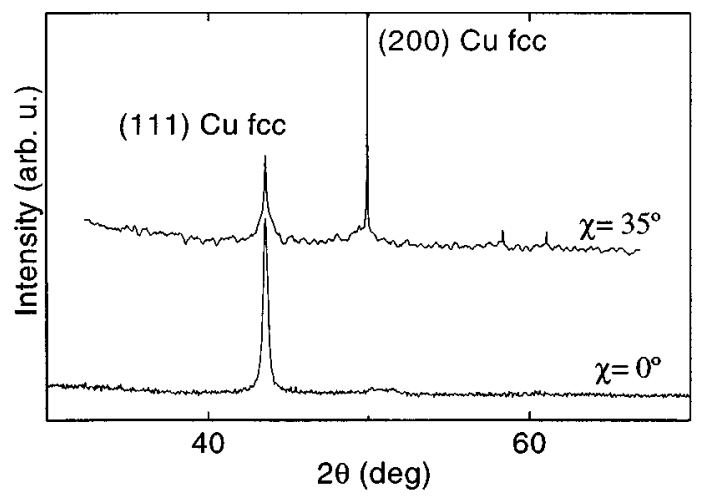

FIG. 2. XRD spectra for a $\mathrm{Co}_{11} \mathrm{Fe}_{6} \mathrm{Cu}_{83}$ as-cast sample at $\chi=0^{\circ}$ and $\chi$ $=35^{\circ}$. 


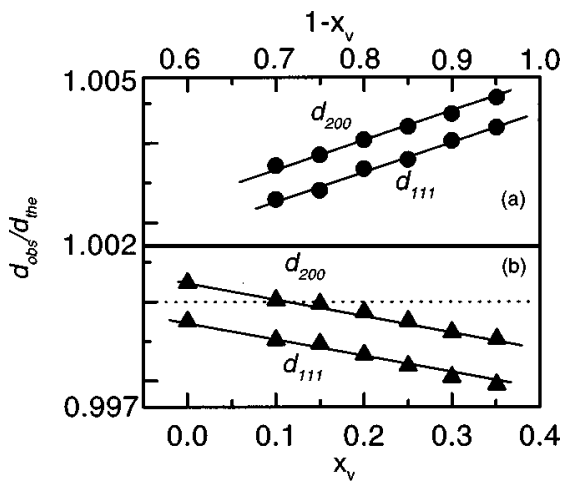

FIG. 3. Interplanar lattice spacing $d_{h k l}$ for the (111) and (200) (a) CoFe and (b) $\mathrm{Cu}$ reflections as a function of the FM concentration for several as-cast samples.

normal to the film plane and stretched in the parallel direction. This is because when atoms are deposited onto the glass substrate, the film cools down and the mean atomic distances tend to shrink, but the glass substrate, with a lower thermal expansion coefficient, avoids film contraction. Thus, the mean in-plane distances are larger than expected, while the perpendicular ones are shorter. Thus, it has been found that the unit cell that best fits the XRD spectra is a rombohedric one with angles of $90.5^{\circ}$ and $89.5^{\circ}$ for all as-cast samples, irrespective of the FM content. By adding $\mathrm{CoFe}$, the lattice spacings of $\mathrm{Cu}$ tend to decrease due to $\mathrm{CoFe}-\mathrm{Cu}$ alloying and MCI strains. However, while the substrate-sample strains are anisotropic, the deformation due to the presence of CoFe are isotropic and equally affects all the $d_{h k l}$ spacings as can be observed in Fig. 3, where the $d_{111}$ and $d_{200}$ versus $x_{v}$ slopes are parallel. With annealing, the spacings evolve to the expected bulk values so the crystal structure goes from a rombohedric to a cubic symmetry. The same happens with the fcc CoFe crystals, for which these slight deformations might be enough to produce a uniaxial magnetoelastic anisotropy as shown in Ref. 6, where an axial deformation of about $0.01 \%$ in a fcc crystal is enough to induce uniaxial anisotropy. This uniaxial anisotropy is perpendicular to the film plane due to the sample texture. Uniaxial anisotropies are found in cubic systems and considered to be caused by the stress which might be produced by a difference in thermal expansion coefficients between film and substrate or by an epitaxial misfit. ${ }^{7}$ Thus, perpendicular anisotropy has also been observed in a variety of fcc thin films grown on a single-crystal substrate, such as fcc PtMnSb grown on $\mathrm{Mn}_{2} \mathrm{O}$ (see for example Ref. 8), where the lattice mismatch between the sample and the substrate is responsible for the perpendicular anisotropy.

Through annealing the microstructure evolves to a higher degree of crystallinity and phase segregation, and strain relaxation occurs. Thus, as particles grow with annealing, peaks shift to their expected bulk values and they become narrower, increasing their intensities (Fig. 1) and the (111) reflection corresponding to the fcc CoFe particles, which is also textured normal to the film, becomes clearly observable. No extra reflections appear for the $\mathrm{Cu}$ phase, so it remains textured along the $\langle 111\rangle$ direction, as the rocking curves confirm. XRD spectra indicate that the size of the $\mathrm{Cu}$

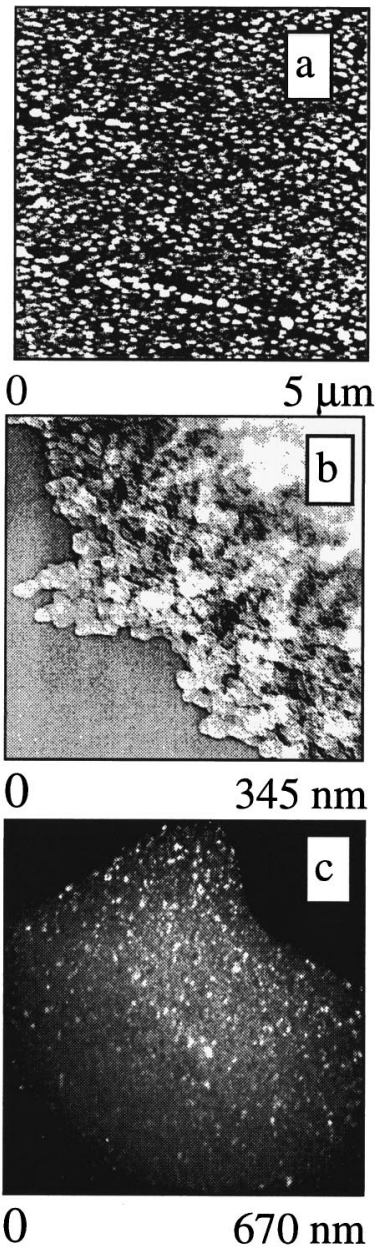

FIG. 4. (a) $\mathrm{AFM}$ image of the surface of a $\mathrm{Co}_{19} \mathrm{Fe}_{8} \mathrm{Cu}_{73}$ as-cast sample, (b) bright field TEM picture of a $\mathrm{Co}_{12} \mathrm{Fe}_{7} \mathrm{Cu}_{81}$ as-cast sample, (c) dark field TEM picture of the CoFe crystals in a $\mathrm{Co}_{12} \mathrm{Fe}_{7} \mathrm{Cu}_{81}$ as-cast sample.

particles varies from 15 to $30 \mathrm{~nm}$ for the as-cast samples and from 25 to $45 \mathrm{~nm}$ for the $750{ }^{\circ} \mathrm{C}$ annealed samples, both depending on the $\mathrm{Cu}$ concentration (the higher the concentration, the larger the particles before and after annealing). The $\mathrm{CoFe}$ particles segregated after soft annealing (at $600^{\circ} \mathrm{C}$ ) have a mean size of $10 \mathrm{~nm}$ irrespective of the $\mathrm{FM}$ concentration, which increases to $30-35 \mathrm{~nm}$ for the $750{ }^{\circ} \mathrm{C}$ annealed samples, depending on the $\mathrm{CoFe}$ concentration. AFM and bright field TEM images show a narrow $\mathrm{Cu}$ particle size distribution in the as-cast samples and a wider one in the annealed ones. Figures 4(a) and 4(b) show an example of these images for as-cast $\mathrm{Co}_{12} \mathrm{Fe}_{7} \mathrm{Cu}_{81}$, from which the granular nature of the samples is observed, being the surface roughness about $30 \pm 5 \mathrm{~nm}$. The granular nature of the samples is also evidenced by dark field TEM; the size of the $\mathrm{Cu}$ and $\mathrm{CoFe}$ particles deduced from this method is consistent with the XRD results. In Fig. 4(c), the dark field of CoFe-rich pseudoamorphous particles is shown, the mean size of the particles is $8+/-5 \mathrm{~nm}$. The matrix surrounding these particles consists of $\mathrm{Cu}$ crystals with $10 \%$ of $\mathrm{CoFe}$ alloyed. This microstructure is of the greatest importance to explain magnetotransport properties. 


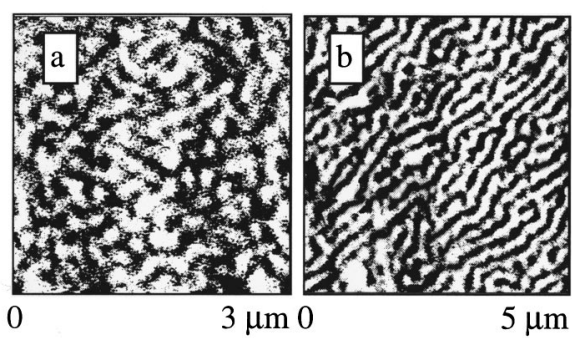

FIG. 5. MFM pictures of a $\mathrm{Co}_{19} \mathrm{Fe}_{8} \mathrm{Cu}_{73}$ as-cast sample and a $\mathrm{Co}_{23} \mathrm{Fe}_{11} \mathrm{Cu}_{66}$ as-cast samples at room temperature and at remanent state.

\section{B. MFM images}

For all as-cast samples with FM content above 20\%, MFM images show long range magnetic domain microstructures (Fig. 5). These MFM images evidence the existence of a magnetic microstructure perpendicular to the film plane: The magnetic moments of neighboring grains tend to be arranged parallel in an out-of-plane direction, leading to stripelike magnetic domains that are themselves aligned antiparallel, which leads to an overall demagnetized state. These magnetic domains, much larger than the particle sizes, are stabilized due to the interplay of: (i) dipolar interactions, which tend to flux closure of neighboring magnetic domains of opposite magnetization, (ii) indirect FM interactions through the matrix, which tend to form large FM domains, and (iii) perpendicular anisotropy, arising from crystal distortion, as observed in $\mathrm{CoFe}-\mathrm{Ag}(\mathrm{Cu})$ granular alloys. ${ }^{9,10} \mathrm{The}$ origin of these terms in the Hamiltonian of the present granular media can be justified as follows. Dipolar interactions are intrinsic to small particle systems. It is suggested that indirect FM exchange among particles takes place through the matrix, since the matrix itself is ferromagnetic for all as-cast samples due to $\mathrm{CoFe}-\mathrm{Cu}$ alloying. The degree of alloying is constant, i.e., the $\mathrm{Cu}$ matrix is $\mathrm{CoFe}$ saturated from the lowest FM composition studied $\left(x_{v} \sim 0.10\right)$. Thus, the stripedlike domains may only appear when the interparticle distance is short enough $\left(x_{v} \geqslant 0.20\right){ }^{11}$ Finally, the origin of the uniaxial perpendicular anisotropy, responsible for the out-ofplane component of the magnetization, is found in the crystal cell deformation. MFM images of the as-cast samples show that the width of the domains increases with increasing the FM content, from about $105 \mathrm{~nm}$ for $x_{v}=0.26$ to $125 \mathrm{~nm}$ for $x_{v}=0.33$. The intensity of the recorded signal and the sharpness of the domains also increase with $x_{v}$, suggesting a larger out-of-plane component of the magnetization, as expected when decreasing the mean distance between the FM particles. However, below $x_{v}=0.20$, MFM images do not show any additional contrast with respect to the AFM images, indicating that no magnetic domains are present since interactions are weaker due to the fact that FM particles are further apart. Through annealing, particles grow leading to the formation of large FM clusters with the magnetic moment lying in the film plane. ${ }^{9,10}$ Hysteresis loops show that the perpendicular anisotropy disappears, due to stress relaxation. Moreover, as the particles segregate, the matrix is no longer FM, and the out-of-plane magnetic microstructure is lost.

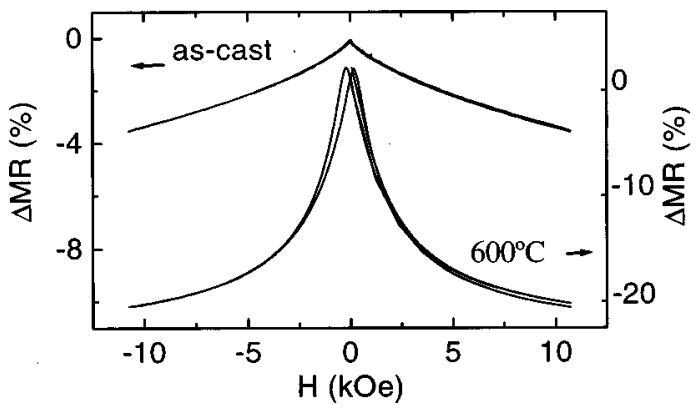

FIG. 6. $\Delta \mathrm{MR}$ vs applied in-plane field in parallel geometry at $20 \mathrm{~K}$ for a $\mathrm{Co}_{11} \mathrm{Fe}_{6} \mathrm{Cu}_{83}$ sample, (a) as-cast and (b) annealed at $600{ }^{\circ} \mathrm{C}$.

\section{Transport properties}

Taking into account the magnetotransport properties, this family of samples can be classified in three different groups. Below $x_{v} \sim 0.20$, annealed samples display the typical features of a granular solid constituted by a random distribution of nanometric $\mathrm{CoFe}$ particles within the $\mathrm{Cu}$ matrix. ${ }^{5,12,13}$ The maximum magnetoresistance observed in this family is about $20 \%$ at low temperature $(T=20 \mathrm{~K}$ and $H=10 \mathrm{kOe}$ ) for an $x_{v}=0.16$ sample annealed at $600^{\circ} \mathrm{C}$. The change in magnetoresistance is defined as,

$$
\Delta \mathrm{MR}=\frac{[R(T, H=0)-R(T, H)]}{R(T, H=0)} .
$$

The curves corresponding to the as-cast samples (Fig. 6) display low values of MR (below 4\%) because CoFe is mainly alloyed with the $\mathrm{Cu}$ matrix, thus the scattering responsible for GMR is not very effective because of the lack of sharp interfaces and the dilution of the magnetic moments in the $\mathrm{Cu}$ matrix. ${ }^{14}$ As a consequence, with annealing MR increases, the material becomes magnetically softer, and hysteresis effects appear due to the growth of relatively large $(>10 \mathrm{~nm})$ CoFe particles (see Fig. 6).

For $x_{v}=0.20-0.30$, MR for the as-cast samples displays complex bimodal behavior and large metastable effects, which are associated with high magnetic correlations ${ }^{10}$ (Fig. 7). The inner peaks correspond to all the irreversible contributions, such as domain wall motion and those arising from granularity (isolated FM particles and uncompensated moments of the antiparallel arrangement), while the outer broad maxima are attributed to the progressive rotation of the magnetic domains structure towards the field axis. There is also a third contribution at intermediate fields arising from the

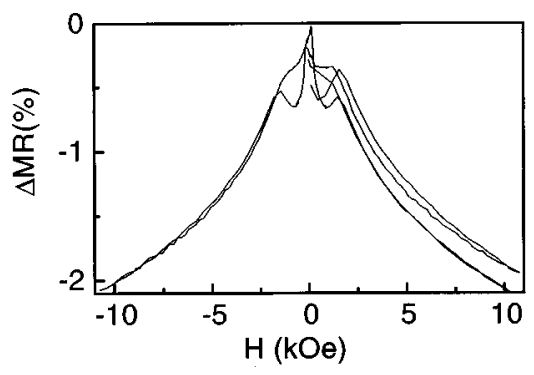

FIG. 7. $\Delta \mathrm{MR}$ vs in-plane applied field in parallel geometry at $20 \mathrm{~K}$ for a $\mathrm{Co}_{22} \mathrm{Fe}_{8} \mathrm{Cu}_{70}$ sample as-cast. 


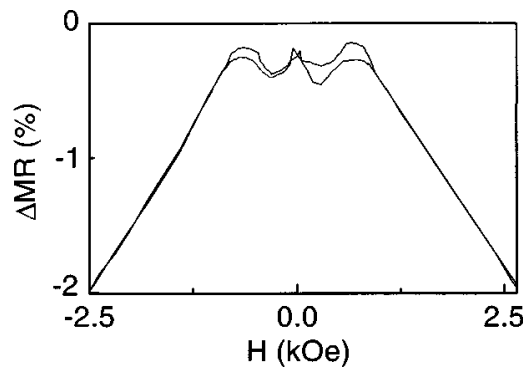

FIG. 8. $\triangle \mathrm{MR}$ vs in-plane applied field in parallel geometry at room temperature for $\mathrm{Co}_{22} \mathrm{Fe}_{8} \mathrm{Cu}_{70}$ as-cast sample.

CoFe alloyed in the matrix, giving place to an AMR, which is responsible for the slight increase in MR at fields of a few hundred Oersteds in the parallel geometry. The high magnetic correlations lead to small values of MR $(\sim 2 \%)$ and anomalous magnetic training behavior in as-cast samples. We use the expression "training behavior" to refer to the fact that the MR curves change as the magnetic field is repeatedly cycled, i.e., each time a $\pm 10 \mathrm{kOe}$ field is applied, the resistivity decreases (Fig. 7). After few loops, training effects disappear and a new (metastable) state is achieved, which remains stable while further measurements are performed. This new metastable state is lost after about $24 \mathrm{~h}$ at zero field and then, the training behavior can be repeated. All these findings reveal the coexistence of highly degenerated remanent states due to magnetic correlations and disorder. The observed decrease in the resistance might be due to the fact that as the magnetic field is cycled, magnetic moments tend to be in plane, forming large in-plane domains leading to a reduction of the electron scattering in the domain walls, as shown in $\mathrm{CoFe}-\mathrm{Ag}(\mathrm{Cu})$ samples. ${ }^{10}$ A more detailed explanation may be found in Ref. 15 .

Stripe-like domains and particles do not show the same thermal behavior because of differences in magnetic size. Thus, while at low temperature the inner peaks of the MR are higher than the outer peaks, the opposite is observed at room temperature (Fig. 8), probably because small magnetic entities tend to become superparamagnetic and no longer participate in the MR, while the outer maxim associated with the antiparallel stripe structure is stable at room temperature, as shown by MFM. As a consequence of the annealing, the characteristic GMR behavior of a granular solid is recovered (Fig. 9). In the samples with lower FM content particles grow slowly with annealing, ${ }^{16}$ then the bimodal behavior remains after soft annealing, while for samples with higher FM

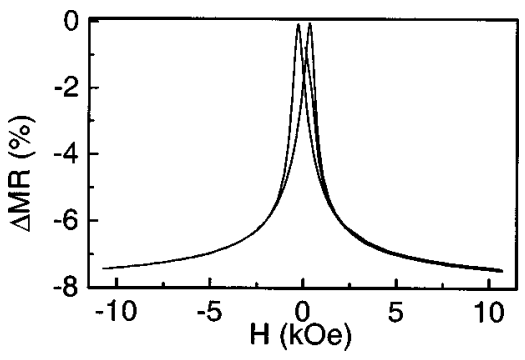

FIG. 9. $\Delta \mathrm{MR}$ vs in-plane applied field in parallel geometry at $20 \mathrm{~K}$ for $\mathrm{Co}_{22} \mathrm{Fe}_{8} \mathrm{Cu}_{70}$ sample annealed at $750{ }^{\circ} \mathrm{C}$.

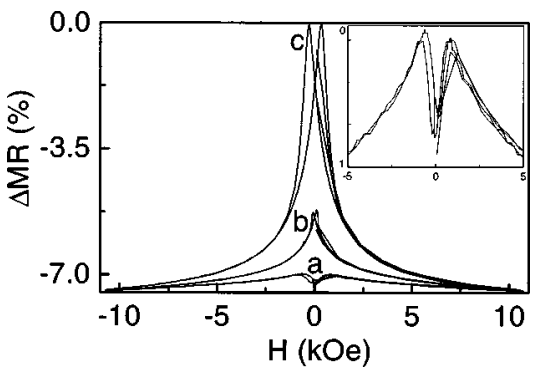

FIG. 10. $\triangle \mathrm{MR}$ vs in-plane applied field in parallel geometry for a $\mathrm{Co}_{23} \mathrm{Fe}_{11} \mathrm{Cu}_{66}$ sample at $20 \mathrm{~K}$ (a) (detail in inset) as-cast, annealed at (b) $600^{\circ} \mathrm{C}$ and (c) $750{ }^{\circ} \mathrm{C}$.

content this behavior rapidly disappears even at soft annealings, due to the precipitation of large particles.

Above $x_{v} \sim 0.30$ (and still below the volume percolation threshold), as-cast samples show both AMR and GMR, as previously observed in Co-Ag alloys. ${ }^{4}$ However, the annealing procedure leads to the complete segregation of the FM content, which results in a transition from the AMR-GMR regime to the normal GMR regime, with a maximum GMR of about 7\% (Fig. 10). Therefore, in the as-cast samples the $\mathrm{CoFe}-\mathrm{Cu}$ alloy is rich enough in FM content to display AMR, as other ferromagnetic alloys do. In fact, both phenomena coexist, e.g., for $\mathrm{Co}_{23} \mathrm{Fe}_{11} \mathrm{Cu}_{66}$, the as-cast sample (inset of Fig. 10) displays AMR at low fields, while GMR dominates above $\sim 1000$ Oe. This sample presents AMR up to 765 Oe with a variation of the resistance about $0.3 \%$, and above 765 Oe GMR dominates, displaying a low value of MR $(0.5 \%)$. AMR is no longer observable after the first annealing $\left(600^{\circ} \mathrm{C}\right)$, and the value of GMR grows to $2.3 \%$. MR increases with annealing up to $4.10 \%$ for $650{ }^{\circ} \mathrm{C}$ and $7.31 \%$ for $750{ }^{\circ} \mathrm{C}$. The bimodal behavior observed in the range from $x_{v}=0.20$ to 0.30 is not detected for larger FM concentrations. However, for the as-cast samples, the inner peak structure is probably hidden by the AMR effect [we note that in some $\mathrm{CoFe}-\mathrm{Ag}(\mathrm{Cu})$ alloys, AMR coexists with the bimodal peak structure].

\section{Magnetic properties}

The magnetization curves for as-cast samples with low FM content $\left(x_{v}<20 \%\right)$ only display hysteresis below about $10 \mathrm{~K}$, showing superparamagnetic behavior at higher temperatures due to the absence of large magnetic particles. Besides, in the as-cast samples with a high FM content $\left(x_{v}\right.$ $>30 \%$ ), hysteresis decreases from 5 to $150 \mathrm{~K}$ (the coercive field $H_{c}$ decreases from 200 to $25 \mathrm{Oe}$ ), and remains almost constant from 150 to $300 \mathrm{~K}\left(H_{c} \sim 25 \mathrm{Oe}\right)$. These facts may indicate the formation of magnetic domains $(>100 \mathrm{~nm})$, due to FM exchange through the FM matrix, which are stable at room temperature, as MFM shows. For samples with $x_{v}$ $=0.20-0.30$, the in-plane magnetization curves for as-cast samples seem to arise from the superimposition of two hysteresis loops: ${ }^{10}$ (i) the inner loop corresponds to all the irreversible contributions, such as the domain wall motion and domain rotation, and other contributions coming from the granularity, such as those corresponding to both isolated magnetic particles and uncompensated moments of the anti- 


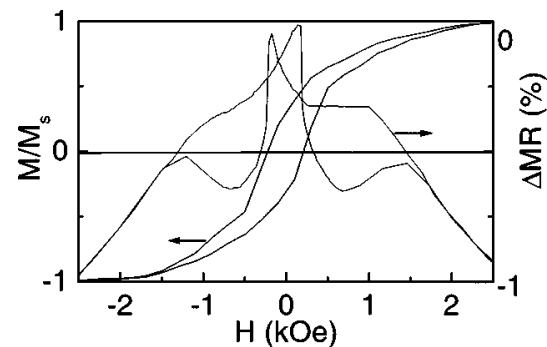

FIG. 11. $\Delta$ MR and hysteresis loop for a $\mathrm{Co}_{22} \mathrm{Fe}_{8} \mathrm{Cu}_{70}$ as-cast sample at $20 \mathrm{~K}$ with the field applied in the plane direction in parallel geometry.

parallel stripe-like domain arrangement, and (ii) the outer loop corresponds to the progressive rotation of the antiparallel domain arrangement towards the field axis, leading to a quasilinear field dependence of the magnetization (Fig. 11). The field dependence of the magnetization curves is consistent with the main features observed in the MR curves (Fig. 11). The inner relative maxima of the MR, occur at the coercive field of the hysteresis loop $(\sim 100 \mathrm{Oe})$ and the outer ones occur at about the field at which irreversibility disappears (1500-2000 Oe). We note that the irreversible contribution (inner loops) is larger for the parallel hysteresis loop than for the perpendicular one, since in the latter, the rotation of the cluster moments towards the field axis is not affected by the random topological distribution of stripe-like domains in the film plane. For $x_{v}>0.30$ hysteresis loops display the same features. No discontinuity suggesting the nucleation of magnetic bubbles is observed in the hysteresis loop for the perpendicular geometry (Fig. 12), in contrast with results for Co thin films,${ }^{17}$ since we are dealing with a granular system so domain inversion does not take place continuously. All these facts suggest that granular alloys displaying long range domain-like structures share some features of both continuous and discontinuous magnetic systems. Through annealing, the magnetic hysteresis and the saturation magnetization increase as $\mathrm{CoFe}$ particles precipitate and grow, and the irreversibility and the squareness of the hysteresis loops increases as the crystals anisotropy evolves from uniaxial to cubic. The bimodal behavior disappears and the maxima of the MR curves occur at $H_{c}$ (about $500 \mathrm{Oe}$ ), having a low thermal dependence.

The remanent-to-saturation magnetization ratio $\left(M_{r} / M_{s}\right)$ increases with annealing, approaching 0.823 at low temperature: the expected value for a cubic symmetry with the easy axis in the $\langle 100\rangle$ direction (Fig. 13). For the

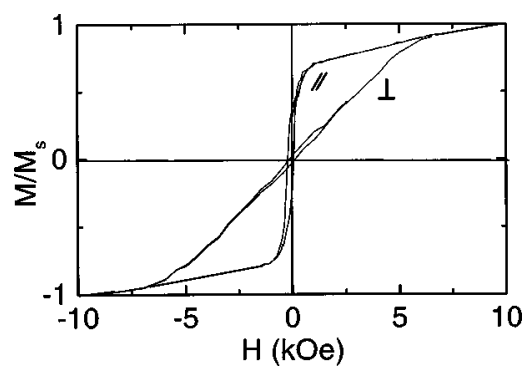

FIG. 12. Parallel and perpendicular normalized hysteresis loops at room temperature for a $\mathrm{Co}_{26} \mathrm{Fe}_{5} \mathrm{Cu}_{69}$ as-cast sample.

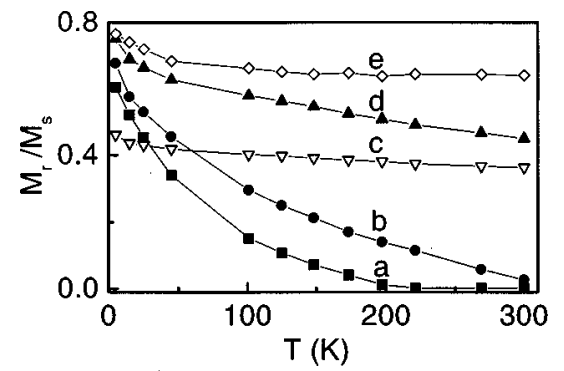

FIG. 13. $M_{r} / M_{s}$ ratio for a $\mathrm{Co}_{12} \mathrm{Fe}_{7} \mathrm{Cu}_{81}$ sample (a) as cast, annealed at (b) $650{ }^{\circ} \mathrm{C}$ and annealed at (c) $750{ }^{\circ} \mathrm{C}$ and for a $\mathrm{Co}_{23} \mathrm{Fe}_{11} \mathrm{Cu}_{66}$ sample (d) as cast and annealed at (e) $750^{\circ} \mathrm{C}$.

as-cast samples, the uniaxial anisotropy coming from the deformed fcc cell leads to a $M_{r} / M_{s}$ value tending to 0.5 at low temperature (Fig. 13), as expected for uniaxial anisotropy. ${ }^{18}$ However, for some as-cast samples, this value is greater than 0.5 , which might be due to the coexistence of axial distorted and cubic fcc crystals. In fact, the deformations near the substrate must be much greater than those far from it, because there is stress relaxation due to plastic deformations (dislocations, vacancies, ... ) as the film grows. Thus, for the as-cast sample with $x_{v}=0.18$, the thickness is $355 \mathrm{~nm}$ and $M_{r} / M_{s}(5 \mathrm{~K})$ is $\sim 0.6$ and for the as-cast sample with $x_{v}$ $=0.33$, the thickness is $240 \mathrm{~nm}$ and $M_{r} / M_{s}(5 \mathrm{~K})$ is $\sim 0.5$. Assuming that the mean blocking temperature, $\left\langle T_{B}\right\rangle$, may be approximated as: $K V=25 k_{B} T_{B}$, where $V$ is the particle volume, and $k_{B}$ the Boltzmann constant, it is found that $T_{B}$ for particles with a diameter of $10 \mathrm{~nm}$ is $46 \mathrm{~K}$. Thus, at room temperature, all the as-cast and soft annealed samples (all these samples present particles with a mean diameter below $10 \mathrm{~nm}$ ) should be superparamagnetic and the $M_{r} / M_{s}$ value should be zero. However, in the samples with high FM content $\left(x_{v}>0.20\right)$, magnetic correlations keep the $M_{r} / M_{s}$ ratio constant with $T$ since these correlations overcome the thermal decrease of $M_{r} / M_{s}$. For samples annealed at $750{ }^{\circ} \mathrm{C}$, $\left\langle T_{B}\right\rangle$ should be about $1000 \mathrm{~K}$ for the sample $x_{v}=0.18$ (mean particle diameter, $28 \mathrm{~nm}$ ) and about $1900 \mathrm{~K}$ for the sample $x_{v}=0.33$ (mean particle diameter, $35 \mathrm{~nm}$ ). Thus, the weak temperature dependence of the $M_{r} / M_{s}$ ratio of the $750{ }^{\circ} \mathrm{C}$ annealed samples has to be attributed to the large size of the FM particles rather than to interparticle interactions.

The orientation of the anisotropy axes in the samples may be calculated using simple energy arguments by comparing parallel and perpendicular hysteresis loops ${ }^{19}$ (Fig. 12). The $\theta$ angle of the resulting uniaxial anisotropy $K$ with respect to the film normal is found through the expressions ${ }^{19}$

$$
\begin{aligned}
& H_{s \|}+H_{s \perp}=4 \pi M_{s}+\frac{2 K}{M_{s}}, \\
& H_{s \|}=\frac{2 K \cos ^{2} \theta}{M_{s}},
\end{aligned}
$$

where $H_{s \|}$ is the parallel saturation field, $H_{s \perp}$ is the perpendicular saturation field, and $M_{s}$ is the saturation magnetization. This procedure is valid for an uniaxial system, but may also be applied to mixtures of uniaxial and cubic systems as explained by Dormann, Fiorani, and Tronc in Ref. 20 with 
TABLE II. Angle of the magnetic anisotropy $(\theta)$ with respect to the film normal and anisotropy constant $(K)$ for different FM contents and annealing temperatures. $M_{s}$ is the saturation magnetization, $H_{s \|}$ is the parallel saturation field and $H_{s \perp}$ is the perpendicular saturation field.

\begin{tabular}{ccccccr}
\hline \hline Sample & $x_{v}$ & $M_{s}\left(\mathrm{emu} / \mathrm{cm}^{3}\right)$ & $H_{s \|}(\mathrm{Oe})$ & $H_{s \perp}(\mathrm{Oe})$ & $K\left(\mathrm{erg} / \mathrm{cm}^{3}\right) 10^{6}$ & $\theta^{\circ}$ \\
\hline $\mathrm{Co}_{7} \mathrm{Fe}_{4} \mathrm{Cu}_{89}$ as-cast & 0.10 & 180 & 2150 & 2250 & 0.19 & 0 \\
$\mathrm{Co}_{12} \mathrm{Fe}_{7} \mathrm{Cu}_{81}$ as-cast & 0.18 & 282 & 1500 & 3500 & 0.20 & 0 \\
$\mathrm{Co}_{12} \mathrm{Fe}_{7} \mathrm{Cu}_{81} 750^{\circ} \mathrm{C}$ & 0.18 & 348 & 2800 & 8200 & 1.6 & 54 \\
$\mathrm{Co}_{22} \mathrm{Fe}_{9} \mathrm{Cu}_{69}$ as-cast & 0.30 & 420 & 1500 & 5400 & 0.34 & 0 \\
$\mathrm{Co}_{22} \mathrm{Fe}_{9} \mathrm{Cu}_{69} 750^{\circ} \mathrm{C}$ & 0.30 & 544 & 2600 & 10000 & 2.39 & 56 \\
$\mathrm{Co}_{23} \mathrm{Fe}_{11} \mathrm{Cu}_{66}$ as-cast & 0.33 & 490 & 980 & 5000 & 0.19 & 0 \\
$\mathrm{Co}_{23} \mathrm{Fe}_{11} \mathrm{Cu}_{66} 600^{\circ} \mathrm{C}$ & 0.33 & 568 & 1500 & 5800 & 0.62 & 34 \\
$\mathrm{Co}_{23} \mathrm{Fe}_{11} \mathrm{Cu}_{66} 650^{\circ} \mathrm{C}$ & 0.33 & 631 & 2300 & 7800 & 1.80 & 52 \\
$\mathrm{Co}_{23} \mathrm{Fe}_{11} \mathrm{Cu}_{66} 750^{\circ} \mathrm{C}$ & 0.33 & 631 & 2700 & 9500 & 2.40 & 54 \\
\hline \hline
\end{tabular}

the particularity that the calculated $K$ corresponds to an effective average of the different uniaxial and cubic (first and second order) contributions. Furthermore, the anisotropy of these systems is the result of the combination of the magnetoelastic, surface, and shape contributions, and the interparticle interactions, in such a manner that the resulting $K$ values can hardly be attributed to one precise origin. The $\theta$ angles obtained as a function of the FM concentration and the annealing temperature are showed in Table II. From Table II, the following conclusions may be drawn: (i) irrespective of the FM content, the magnetic anisotropy stays perpendicular to the film plane $(\theta=0)$, as the crystal structure of the as-cast samples remains basically the same, (ii) when particles become larger through annealing, and the crystals evolve from distorted cubes to fcc cells, the angle of the magnetic anisotropy moves from $0^{\circ}$ towards $54.7^{\circ}$ (angle between the $\langle 111\rangle$ and $\langle 100\rangle$ cube directions), which is the expected value for an (111) fcc textured crystal, (iii) $K$ for as-cast samples is almost constant (experimental values vary between $0.2 \times 10^{6}$ and $0.4 \times 10^{6} \mathrm{erg} / \mathrm{cm}^{3}$ ) since the crystal deformation is independent of $x_{v}$, and (iv) with annealing $M_{s}$ increases and the saturation fields also increase leading to larger values of $K$. The values corresponding to the cubic case are larger than those corresponding to the uniaxial case, and larger than $0.8 \times 10^{6} \mathrm{erg} / \mathrm{cm}^{3}$, which corresponds to a noninteracting distribution of Co fcc FM particles. ${ }^{21}$ This may be due to high magnetic interactions in the CoFe aggregates resulting from the annealing procedure (direct exchange). However, it is difficult to compare these situations since in the as-cast samples there is a ferromagnetic matrix with diluted magnetic moments and slight uniaxial deformation, while after annealing the matrix is no longer FM and all the $\mathrm{CoFe}$ is well precipitated and crystallized in large (up to $100 \mathrm{~nm}$ ) particle clusters.

\section{CONCLUSIONS}

As the FM content is increased, transport properties evolve from GMR to AMR, with an intermediate region in which high magnetic correlations strongly affect the magnetotransport properties, leading to low values of GMR. For the samples with $x_{v}<0.20$, GMR values of $20 \%(T=20 \mathrm{~K}$, $H=10 \mathrm{kOe})$ are found after moderate annealing. When the FM concentration increases $\left(x_{v}=0.20-0.30\right)$ magnetic interactions become stronger and MR decreases. When $x_{v}$
$>0.30$, the $\mathrm{CoFe}-\mathrm{Cu}$ alloy present in the as-cast samples displays AMR behavior. Through annealing $\mathrm{CoFe}$ particles grow and normal GMR with moderate strength $(7 \%$ at $T=20 \mathrm{~K}$ and $H=10 \mathrm{kOe}$, for $x_{v}=0.33$ ) is observed. Structural and magnetic characterization is consistent with these results. In the as-cast samples, $\mathrm{CoFe}$ particles, $\mathrm{Cu}$ particles, and a $\mathrm{FM} \mathrm{CoFeCu}$ alloy coexist setting up complex dipolar and exchange interactions. Furthermore, due to residual glass substrate-film stress, there is a perpendicular magnetic uniaxial anisotropy. When the $\mathrm{CoFe}$ particles dispersed in the $\mathrm{Cu}(\mathrm{CoFe})$ matrix are close enough $\left(x_{v}>0.20\right)$, and below the percolation threshold, stripe-like magnetic domains stabilize ${ }^{10}$ The annealing procedure segregates the $\mathrm{CoFe}$ alloyed in the matrix, the particles grow and become more separated, and the crystal strains relax. Therefore, the exchange interactions through the matrix and the uniaxial anisotropy disappear and the out-of-plane magnetic domain structures are lost. A variety of ferromagnetic/nonmagnetic granular alloys based on Co display this complex magnetic and transport behavior, the crossover FM contents within those different regimes being the only difference among them, since those values depend on the relative immiscibility of the components of each particular granular alloy. ${ }^{9}$ A detailed study of the $\mathrm{FeNi}-\mathrm{Ag}$ and $\mathrm{CoFe}-\mathrm{Ag}(\mathrm{Cu})$ granular alloys also showed similar features.

\section{ACKNOWLEDGMENTS}

The authors would like to thank Dr. M. L. Watson for supplying the facilities to synthesize the samples. Financial support of both the Spanish CICYT through the MAT-970404 project and the Catalonian CIRIT through the SGR119 project are largely recognized. The Spanish-British Joint Action HB 1996-0066 is also acknowledged.

${ }^{1}$ A. E. Berkowitz et al., Phys. Rev. Lett. 68, 3745 (1992); J. Q. Xiao, J. S. Hiang, and C. Chien, ibid. 68, 3749 (1992).

${ }^{2}$ R. E. Camley and J. Barnes, Phys. Rev. Lett. 63, 644 (1989); P. M. Levy, S. H. Zhang, and A. Fert, ibid. 65, 1643 (1990).

${ }^{3}$ T. R. McGuire and R. I. Potter, IEEE Trans. Magn. 11, 1018 (1975).

${ }^{4}$ J. A. Mendes et al., J. Appl. Phys. 81, 5208 (1997).

${ }^{5}$ S. R. Teixeira et al., J. Phys.: Condens. Matter 6, 5545 (1994).

${ }^{6}$ C. H. Lee, Hui He, F. J. Lamelas, W. Vavra, C. Uher, and R. Clarke, Phys. Rev. B 42, 1066 (1990).

${ }^{7}$ S. Chikazumi, Physics of Magnetism (R. E. Krieger, Malabar, FL, 1964).

${ }^{8}$ R. Carey et al., Seventh Intermag-MMM Conference, San Francisco, 1998. 
${ }^{9}$ X. Batlle, V. Franco, A. Labarta, M. L. Watson, and K. O'Grady, Appl. Phys. Lett. 70, 132 (1997).

${ }^{10}$ V. Franco, X. Batlle, A. Labarta, M. L. Watson, and K. O'Grady, J. Appl. Phys. 81, 4593 (1997); V. Franco, X. Batlle, A. Labarta, A. Valencia, F. Sandiumenge, M. L. Watson, and K. O'Grady, IEEE Trans. Magn. 34, 912 (1998)

${ }^{11}$ V. Franco, X. Batlle, and A. Labarta Phys. Rev. B (submitted).

${ }^{12}$ A. Milner, A. Gerber, B. Groisman, M. Karpovsky, and A. Gladkikh, Phys. Rev. Lett. 76, 475 (1996).

${ }^{13}$ H. Sato, Y. Kobayashi, Y. Aoki, and H. Yamamoto, J. Phys.: Condens. Matter 7, 7053 (1995)

${ }^{14}$ K. Ounadjela et al., Phys. Rev. B 17, 12252 (1996).
${ }^{15}$ V. Franco, X. Batlle, and A. Labarta, J. Magn. Magn. Mater. 196-197, 465 (1999).

${ }^{16}$ F. Badia, X. Batlle, A. Labarta, M. L. Watson, A. B. Johnston, and J. N. Chapman, J. Appl. Phys. 82, 677 (1997).

${ }^{17}$ J. F. Gregg et al., Phys. Rev. Lett. 77, 1580 (1996).

${ }^{18}$ E. C. Stoner and E. P. Wohlfarth, Philos. Trans. R. Soc. London, Ser. A 240, 599 (1948).

${ }^{19}$ J. Q. Xiao, C. L. Chien, and A. Gavrin, J. Appl. Phys. 79, 5309 (1997).

${ }^{20}$ J. L. Dormann, D. Fiorani, and E. Tronc, Adv. Chem. Phys. 98, 283 (1997).

${ }^{21}$ D. S Rodbell, J. Phys. Soc. Jpn. 17, 313 (1961). 